\title{
Special issue on machine learning applications for self-organized wireless sensor networks
}

\author{
Mohamed Elhoseny $^{1} \cdot$ Xiaohui Yuan ${ }^{2} \cdot$ M. Gunasekaran ${ }^{3}$
}

Published online: 11 December 2018

(C) Springer-Verlag London Ltd., part of Springer Nature 2018

This is a special issue of the Neural Computing and Applications (NCAA) journal, covering the machine learning techniques for the smart and the sensor-based applications. Over the last decade, there has been an increasing interest in research of sensors and wireless networks. The interconnection of embedded devices is expected in many systems including mobility, intelligent transportation, and smart cities. It is being promoted by the software engineering community to use network-based machine learning systems as the adequate solution to handle the current requirements of complex big data processing problems that demand distribution, flexibility, and robustness. However, machine learning research has been focused mainly in the traditional network problems with "structure" (images, audio, video), and not in the smart and modern ad hoc or wireless networks problems, making the data processing in such systems a big challenge. In this special issue, we show how machine learning algorithms and novel intelligent techniques may be used to address the open problems in most of the sensor-based applications including Internet of Things (IoT), self-organized wireless sensor networks and intelligent data aggregation and routing models. Because neural computing plays an important role in machine learning, the Special Issue on Machine Learning Applications in Sensor-based environments published on Neural Computing and Applications

M. Gunasekaran

gmanogaran@ucdavis.edu

Mohamed Elhoseny

Mohamed_elhoseny@mans.edu.eg

Xiaohui Yuan

xiaohui.yuan@unt.edu

1 Faculty of Computer and Information Sciences, Mansoura University, Mansoura, Egypt

2 Department of Computer Science and Engineering, University of North Texas, Denton, TX, USA

3 University of California, Davis, CA, USA can play an important role by providing a venue for academics as well as industrial practitioners to disseminate their results in a peer-reviewed environment.

The accepted papers explore diverse modeling approaches and computational intelligence techniques including Multilayer Perceptron, Recurrent Neural Networks, Deep Convolutional Neural Networks, Data Fusion of Multisensors, Fuzzy Logic and Support Vector Machines. A number of novel methods and approaches are proposed and applied to a wide range of real-world problems such as Machine Learning for Health care, IoT-based Agriculture, Big data Processing, Resource Sharing, Real-time Monitoring, and site selection for construction. More specifically, in the paper "Research on data fusion of multisensors based on fuzzy preference relations," the authors investigate the data fusion in multi-sensors applications. In order to improve the accuracy of fusion algorithm, an adaptive weighted algorithm based on fuzzy preference relations is proposed. The degree of preference between signals is represented by introducing the improved logsig function, and then the weight is calculated by fuzzy preference relations. Moreover, the paper "Adapting Weather Conditions Based IoT Enabled Smart Irrigation Technique in Precision Agriculture Mechanisms." This work relies on independent IoT-enabled Wireless Sensor Network (WSN) framework consisting of soil moisture (MC) probe, soil temperature measuring device, environmental temperature sensor, environmental humidity sensing device, $\mathrm{CO}_{2}$ sensor, daylight intensity device (Light Dependent Resistor) to acquire real-time farm information through multi-point measurement. The projected observance technique consists of all standalone IoT-enabled WSN nodes used for timely data acquisitions and storage of agriculture information.

Another example is the paper "A Novel Recommender System Based on FFT With Machine Learning for Predicting and Identifying Heart Diseases." An efficient medical recommendation system has been proposed in this paper, namely Fourier Transformation-based Heart Disease Prediction System (FTHDPS) by using Fourier 
transformation and machine learning technique to predict the chronic heart diseases effectively. Here, the input sequences are relying on the patient's time series details or data, which are crumbled by Fourier transformation for extracting the frequency information.
Finally, we hope that the readers will find all papers in this special issue interesting and useful. The Guest editors wish to thank all the authors and reviewers that contributed to this special issue, and to the Editor-in-Chief and editorial office of the Neural Computing and Application journal for their support. 